\title{
GI highlights from the literature
}

\author{
Mairi H McLean, Education editor
}

\section{BASIC SCIENCE \\ Genetic classification of colorectal cancer subtypes}

\begin{abstract}
- Guinney J, Dienstmann R, Wang X, et al. The consensus molecular subtypes of colorectal cancer. Nat Med 2015;21:1350-6.
\end{abstract}

Cancers display heterogeneity, and thus personalised cancer therapy has the potential to improve patient outcome. Guinney and colleagues developed a consortium with independent analysis of large-scale transcriptome data using independent groups to generate a network of gene pathways that can be ascribed to specific, hitherto unknown colorectal cancer (CRC) subtypes. Whole-genome gene-expression data were used from 18 CRC datasets and a cohort size of 4151 patients. Cancer phenotype was taken into account, and the data were normalised to ensure a comparable form. Statistical analysis generated network plots with each patient representing a node. Each cluster of nodes was investigated for common genomic aberrations and epigenetic and phenotypic features. Each cluster was termed a consensus molecular subtype (CMS). Four CMS were discovered. Approximately $20 \%$ of patients' data did not cluster into any subtype. CMS1 showed a gene expression consistent with strong Th1 immune response and a genetic profile consistent with microsatellite instability; BRAF mutations, a mutator phenotype and a high methylation phenotype. CMS2 (the most frequent subtype) demonstrated a canonical subtype with high WNT signalling and MYC activation along with genes associated with the cell cycle. CMS3 gene expression displayed metabolism deregulation and a high incidence of KRAS mutations. CMS4 showed a more stroma-cell-related gene expression, suggesting association with epithelial-mesenchymal transition, along with high angiogenic gene expression. CMS1 appeared more frequently in females with right-sided CRC and advanced disease, reflected by poor survival. CMS2 were mainly left sided. CMS4 cancers appeared to present with the most advanced disease and displayed the worst survival of all subtypes. It would have been interesting to see the clinical information of the non-consensus patients. This article displays the power of collaborative research, and using independent data mining that is shared and collated has yielded several CRC subtypes that were previously not classified by anything other than APC mutations and clinical features. Further work on these subtypes may yield better personalised therapeutic approaches.

\section{Exploring the cell origin of hepatocellular cancer}

- Mu X, Español-Suñer R, Mederacke I, et al. Hepatocellular carcinoma originates from hepatocytes and not from the progenitor/biliary compartment. J Clin Invest 2015;125:3891903.

One of the distinctive features of hepatocytes is the capability of undergoing indefinite replication. This underlies the virtually unlimited regenerative potential of the liver. This suggests that hepatocytes represent the cell of origin of hepatocellular carcinoma (HCC). However, in most organs, including the intestine, cancers originate from the stem or progenitor compartment. Understanding this is a current and controversial topic in liver carcinogenesis. Previous studies have shown that HCCs can develop from cells of the biliary compartment or from liver progenitor cells (LPC) after forced oncogene expression; this is confirmed by the fact that many HCCs exhibit a progenitor signature, such as the expression of AFP, K19 and A6. Mu and colleagues addressed the issue of the origin of HCC by tracing the fate of selectively labelled cells of the LPC/biliary compartment or hepatocytes during the process of hepatocarcinogenesis in different murine models of genotoxic or genetic tumour induction. By assessing the expression of cell-specific reporter genes, the authors found that HCCs developed solely from hepatocytes, but never from cells of the progenitor or biliary compartment. This data strengthen the notion that HCC arises exclusively from hepatocytes, which should be considered as a fully differentiated cell type with intact stem-cell properties. The fact that some HCCs express a progenitor marker signature is thus likely to reflect the high plasticity of hepatocytes and their ability to undergo dedifferentiation, rather than their origin from the progenitor cell compartment.

\section{The role of miRNA-214 in colitis-associated CRC}

- Polytarchou C, Hommes DW, Palumbo T, et al. MicroRNA214 Is Associated with progression of ulcerative colitis, and inhibition reduces development of colitis and colitis-associated cancer in mice. Gastro 2015;149:981-92.

Current guidelines outline colonoscopic surveillance protocols to identify progression from UC to colitis-associated colorectal cancer (CAC). The molecular signature of this disease progression is not fully understood. Micro-RNAs (miRNAs) are small non-coding RNAs that regulate gene expression, and have been linked to cancer progression at a number of body sites. Here, the authors explore the role of these miRNAs in IBD and progression to CAC. Initial in vitro functional analysis of the comprehensive human microRNAome in colonocytes demonstrated that there are several miRNA candidates requiring further scrutiny. Further analysis in colonoscopic biopsies revealed a profound increased expression of epithelial cell miR-214 in UC, correlated to disease activity and chronicity through time from diagnosis. Novel methodology involving prediction software and high throughput expression analysis identified effectors and regulatory pathways of miR-214 expression in the colon. Increased miR-214 was identified in CAC compared with sporadic cancer and normal controls. Subsequent gain-of-function or loss-of-function studies showed expression of miR-214 can influence epithelial cell behaviour and exposed the oncogenic properties of this miRNA. Chemical inhibition of miR-214 with intracolonic antisense miR-214 attenuated disease activity in a murine model of IBD and crucially reduced tumour size and burden in a chemically induced model of murine CAC. Overall, this study has revealed a novel molecular pathway involved in development of CAC and this could be targeted therapeutically to treat colonic inflammation and disrupt progression to malignancy.

\section{CLINICAL PRACTICE Chemoprevention of CRC with calcium and vitamin D supplementation?}

Baron JA, Barry EL, Mott LA, et al. A trial of calcium and vitamin D for the prevention of colorectal adenomas. N Engl J Med 2015;373:1519-30.

The reputed benefits of calcium and vitamin D supplementation include antineoplastic effects in the GI tract. Newly published data from a large, randomised, placebo-controlled study 
from the USA casts a shadow over previously published epidemiological and preclinical data suggesting that supplementation with these agents can reduce risk of CRC. Reporting in the New England Journal of Medicine, this study found no benefit from daily supplements of vitamin $\mathrm{D}_{3}$ (1000 IU), calcium $(1200 \mathrm{mg})$, or both in the prevention of adenoma recurrence over a period of 3-5 years. Over 2200 patients aged 4575 years were recruited following colonoscopy at which all adenomatous polyps were completely removed and with no further polyps remaining in the colon. Randomised to receive single supplementation with calcium or vitamin $\mathrm{D}_{3}$, combined supplementation or placebo, patients were followed up at 6 monthly intervals until repeat colonoscopy at either 3 or 5 years. $45.7 \%$ individuals taking combined supplements developed recurrent adenomas compared with $48.2 \%$ taking neither. $45.3 \%$ taking calcium supplements were found to have further adenomas compared with $47.6 \%$ who did not take calcium and rates of adenoma recurrence in those taking vitamin $\mathrm{D}_{3}$ were identical to those who did not receive vitamin $\mathrm{D}_{3}$ supplements. These unexpected results will leave many researchers scratching their heads. Perhaps longer-term supplementation and follow-up is required for a beneficial effect to be seen or maybe higher doses of vitamin $\mathrm{D}_{3}$ are needed? Or could it be that the effect of calcium and vitamin $\mathrm{D}_{3}$ occurs at a later stage of cancer development, by influencing the rate of progression from adenoma to carcinoma?

\section{Stem cells for perianal Crohn's disease_promising data}

- Molendijk I, Bert BA, Roelofs H, et al. Allogenic bone marrow-derived mesenchymal stromal cells promote healing of refractory perianal fistulas in patients with Crohn's disease. Gastro 2015;149:918-27.

Perianal Crohn's disease (CD) is one of the most destructive phenotypes of the disease with a massive impact on patients' quality of life. Furthermore, the complicated treatment algorithms advised by national guidelines, coupled with the often disappointing results of biological therapies in this cohort, highlight the massive unmet need for successful therapies in these patients. Mesenchymal stem cells (MSCs) have potent antiinflammatory and immunomodulatory properties. In addition, they are involved in tissue repair making them a potentially attractive therapy for perianal fistulae. Molendijk and colleagues recently published a small randomised phase II study investigating whether local injection of bone-marrow-derived MSCs from healthy donors could lead to fistula healing. Twenty-one patients with refractory perianal CD with non-complex fistulae were randomised to one of three doses of MSCs or placebo. All injections were carried out under general anaesthesia. The primary outcome measure was fistula healing as assessed by blinded physician examination of the external opening and blinded MRI pelvis imaging reporting. No safety signals were noted up to 24 weeks. The patients receiving the lower dose of MSCs $\left(3 \times 10^{7}\right)$ showed the highest rate of response as compared with placebo with $80 \%$ healing at weeks 6 and $12,9(p=0.08)$, and these effects were stable through to week 24 . While these data are promising, the optimal dose and type of MCSs, patient characteristics (especially previous medical therapies), mechanism of action of MSCs, best route of administration and long-term outcomes are far from clear, and future larger phase III studies are eagerly awaited to clarify these issues.

\section{Management of patients with Barrett's oesophagus and low-grade dysplasia}

- Small AJ, Araujo JL, Leggett CL. Radiofrequency ablation is associated with decreased neoplastic progression in patients with Barrett's esophagus and confirmed low-grade dysplasia. Gastro 2015;149:567-76.

Barrett's oesophagus (BO) is a recognised premalignant precursor to oesophageal adenocarcinoma (OAC). Only a small proportion of patients with $\mathrm{BO}$ will progress towards malignancy and there is currently no definitive biomarker to aid identification of high-risk individuals. Current guidelines suggest interval surveillance endoscopy plus mucosal biopsies to detect progression. How should we manage patients that do progress to dysplasia? It is accepted that endoscopic ablation of high-grade dysplasia (HGD) reduces risk of progression to malignancy. The management of $\mathrm{BO}$ associated low-grade dysplasia (LGD) is more contentious. Guidelines suggest frequent endoscopic assessment every 6-12 months to detect progression of LGD, but a recent trial suggests radiofrequency ablation (RFA) is superior at reducing risk. In this paper, Small and colleagues performed a retrospective analysis of patients with $\mathrm{BE}$ +LGD who underwent RFA $(n=45)$ or surveillance endoscopy $(n=125)$, with average follow-up of approximately 850 days, within three US centres. A total of 21 and 56 patients with more than 3 years' follow-up were included in these two groups, respectively. Overall, the annual rate of progression to the endpoint of combined HGD/OAC was $6.6 \%$ in the endoscopic surveillance group. This was significantly reduced to $0.77 \%$ in those who received RFA (adjusted HR $=0.06 ; 95 \%$ CI 0.008 to 0.48 ). The analysis also identified mucosal nodularity and multifocal dysplasia as independent risk factors of progression from LGD to OAC. A crucial part of this management algorithm was expert GI histopathology diagnosis of LGD. Despite its limitations and potential for selection bias, this study suggests that RFA is a superior management strategy for patients with $\mathrm{BO}+\mathrm{LGD}$, in keeping with recent European trial data.

\section{Reviewers}

Dr Stuart McDonald, Dr Enrico De Toni, Dr Mairi McLean, Dr Jonathan MacDonald, Dr Dan Gaya

\section{Journals reviewed}

Nature Medicine, Journal of Clinical Investigation, Gastroenterology, New England Journal of Medicine.

Provenance and peer review Not commissioned; internally peer reviewed.

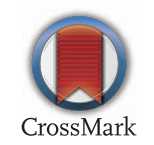

To cite McLean MH. Gut 2016;65:179-180. 